\title{
Finite Element Analysis of Cogging Torque in Low Speed Permanent Magnets Wind Generators
}

\author{
T. Tudorache ${ }^{1}$, L. Melcescu $^{1}$, M. Popescu ${ }^{2}$, M Cistelecan $^{2}$ \\ ${ }^{1}$ University POLITEHNICA of Bucharest, Electrical Engineering Faculty, Electrical \\ Machines Dept., 313 Splaiul Independentei, 060042, Sect. 6, Bucharest (Romania), \\ Phone/Fax number: +0040 21 3197969, e-mail: tudorach@ amotion.pub.ro, leonard@ amotion.pub.ro \\ ${ }^{2}$ Research Institute for Electrical Machines (ICPE-ME), 45 Tudor Vladimirescu, Bucharest, \\ (Romania), Phone number: +0040 21 4100704, e-mail: pd_mihail@yahoo.com, mciste@yahoo.com
}

\begin{abstract}
This paper deals with the finite element estimation of cogging torque oscillations that occur in multipole permanent magnets synchronous wind generators.

The numerical analysis is carried out using a 2D plane-parallel finite element model of the machine and afterward a more elaborated 3D model able to take into account the skewing of the stator slots. The numerical results are discussed and compared with the experimental measurements.

This paper presents also the influence of the magnetization characteristics of stator and rotor cores as well as the influence of the magnetization orientation of the permanent magnets of the generator on the cogging torque values.
\end{abstract}

\section{Keywords}

Finite element analysis, cogging torque.

\section{Introduction}

The increasing global demand of energy, the continuous reduction of fossil fuel resources and the growing concern for environment pollution drew the attention of specialists to new electrical energy production technologies using renewable sources, such as wind energy, solar energy, etc. Among the non-conventional renewable energy sources, wind energy presents the largest potential of conversion into electrical energy, being able in the future to ensure a large part of all the energy need of the planet.

A key role in the conversion chain of wind energy into electricity is played by the electrical generator. From the variety of electrical machines that can be used as generators in wind applications a privileged position is occupied by the directly driven multi-pole permanent magnet synchronous generator [1]-[4]. This type of converter has a large number of poles and can generate electricity at low speeds by operating directly mounted on the main shaft of the wind turbine. This feature is very attractive and permits the simplification of the wind energy conversion system by removing the classical gearbox that typically represents a source of supplementary losses and requires periodical maintenance.

A special attention in the design of the multi-pole permanent magnet synchronous generators should be paid to the reduction of cogging torque oscillations that appear due to the mutual attraction between rotor and stator magnetic armatures of the machine. In this context a proper design of the generator that permits to start the wind turbine at reduced wind speeds of about $2.5-3 \mathrm{~m} / \mathrm{sec}$. supposes to keep the cogging torque values in the range of $1.5-2.5 \%$ out the rated torque [4]. These constraints require reliable finite element based numerical models of the machine able to deal with the delicate aspect of the cogging torque evaluation.

The physical support for this study is represented by a synchronous generator designed and built by the Research Institute for Electrical Machines (ICPE-ME), Bucharest, Romania that is characterized by the main data: rated power $7.5 \mathrm{~kW}$, rated speed $200 \mathrm{rpm}, 36$ stator slots and 26 rotor poles, lamination stack length $140 \mathrm{~mm}$ with an inclination angle of stator slots of $8.4^{\circ}$, airgap thickness $0.85 \mathrm{~mm}$. The permanent magnets are mounted on the outer rotor surface and are unidirectionally magnetized.

\section{2D plane-parallel model of the machine}

The 2D plane-parallel finite element model of the machine equipped with unidirectional magnetized permanent magnets, does not take into account the skewing of stator stack of laminations.

The 2D FEM computation domain is represented by a cross section through the entire machine, no symmetries being taken into account, Fig. 1. The governing differential equation of the magneto-static field is:

$$
\nabla \times[(1 / \mu) \cdot \nabla \times \mathbf{A}]=\nabla \times\left[(1 / \mu) \cdot \mathbf{B}_{\mathbf{r}}\right],
$$

where A stands for the magnetic vector potential, $\mu$ for magnetic permeability of magnetic cores, $\mathbf{B}_{\mathbf{r}}$ for remnant magnetization of permanent magnets. 
The finite element discretization of the computation domain consists of 43712 second order elements of triangle shape with smaller size in the airgap region where the most part of the magnetic energy is located, Fig. 2.

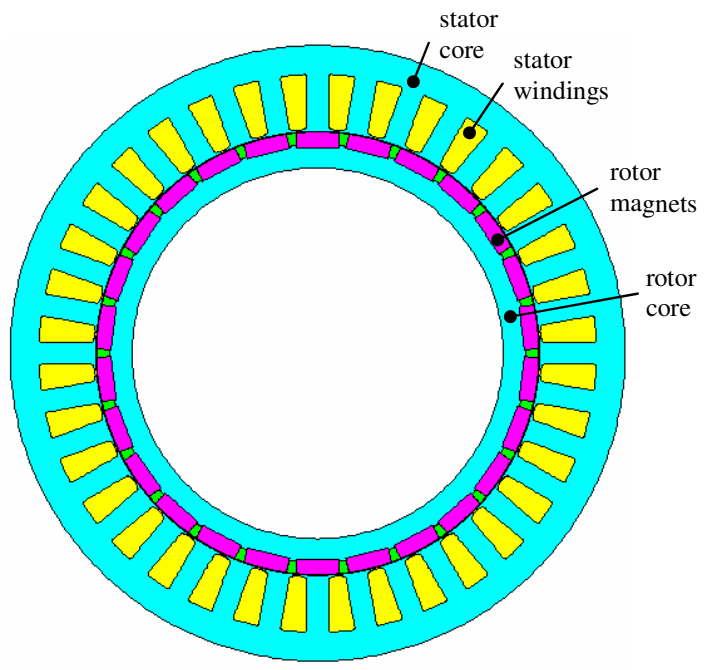

Fig. 1. 2D FEM computation domain.

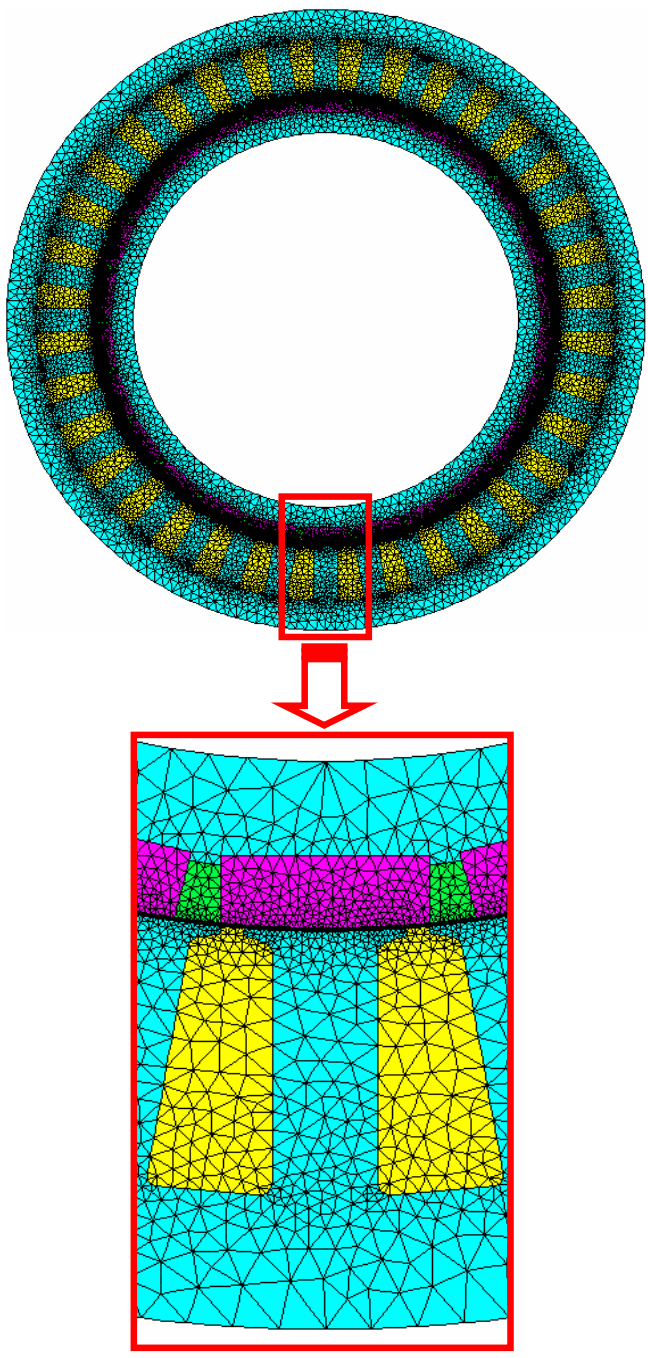

Fig. 2. Mesh of the 2D computation domain.
In order to increase the accuracy of the cogging torque computation results the airgap region is meshed using three layers of finite elements. Our experience reveals that using a single layer of finite elements in the airgap region of the studied machine may lead to results inaccuracy.

The stator and rotor laminations are made of soft magnetic material characterized by saturation magnetization $\mathrm{B}_{\mathrm{s}}=1.8 \mathrm{~T}$ and initial relative magnetic permeability $\mu_{\mathrm{r}}=500$. The permanent magnets are made of $\mathrm{NdFeB}$ with $\mu_{\mathrm{r}}=1.1$, and remnant magnetic flux density $\mathrm{B}_{\mathrm{r}}=1.0446 \mathrm{~T}$.

By solving the $2 \mathrm{D}$ magneto-static field problem for a given relative stator/rotor position using the Flux professional software package, we obtained the magnetic field lines presented in Fig. 3, and the chart of the magnetic flux density in the computation domain shown in Fig. 4.

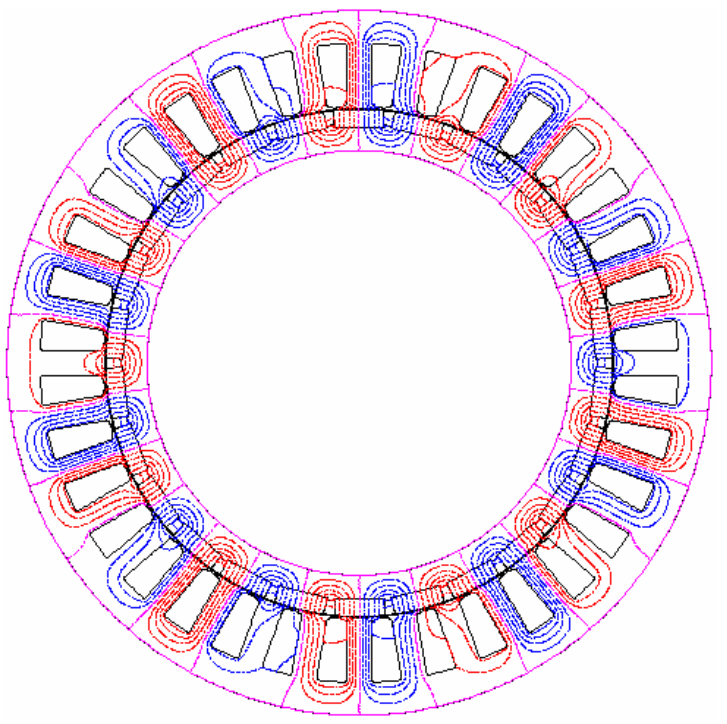

Fig. 3. Magnetic field lines.

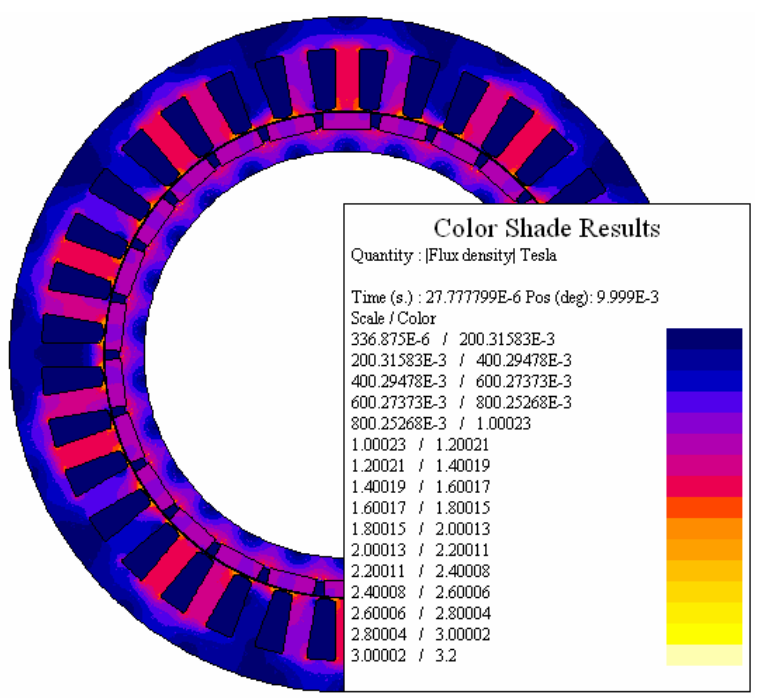

Fig. 4. Chart of the magnetic flux density. 
By solving the 2D magneto-static field problem for several successive relative stator/rotor positions we obtain the cogging torque oscillations by using the Virtual Works Method, Fig. 5.

Studying the numerical results in Fig. 5 we can notice important values of the cogging torque that can affect the effective operation of the synchronous generator for wind applications.

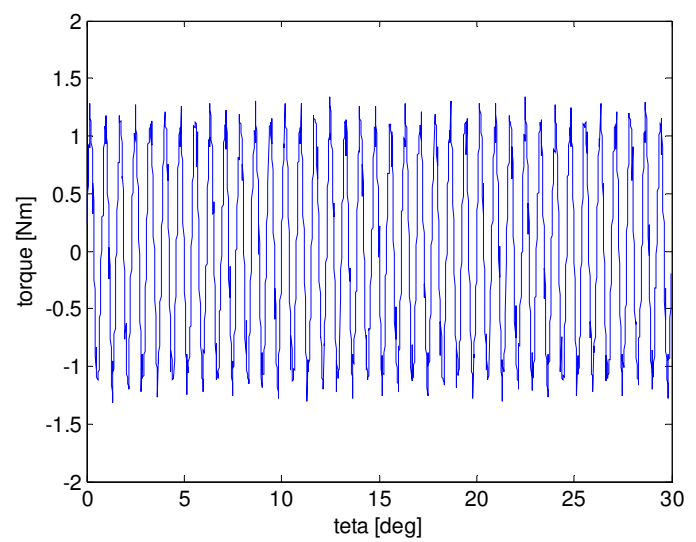

Fig. 5. Cogging torque versus rotor position (2D planeparallel approach, unidirectional magnetization).

\section{Influence of magnetization orientation of permanent magnets}

Permanent magnets used as excitation source in case of synchronous generators can be magnetized in several ways such as: unidirectional, radial, orthoradial etc. Unlike the previous section where the magnetization orientation of permanent magnets was considered unidirectional, here we studied the effect of the radial orientation of the permanent magnets on the cogging torque oscillations.

The numerical approach used for this analysis consists of a 2D plane-parallel FEM model of the machine similar to the one used in the previous section.

The numerical results obtained by Flux simulations prove that the cogging torque values of the generator with radial magnetization of the permanent magnets, Fig. 6, are smaller than the values corresponding to the unidirectional magnetization shown in Fig. 5.

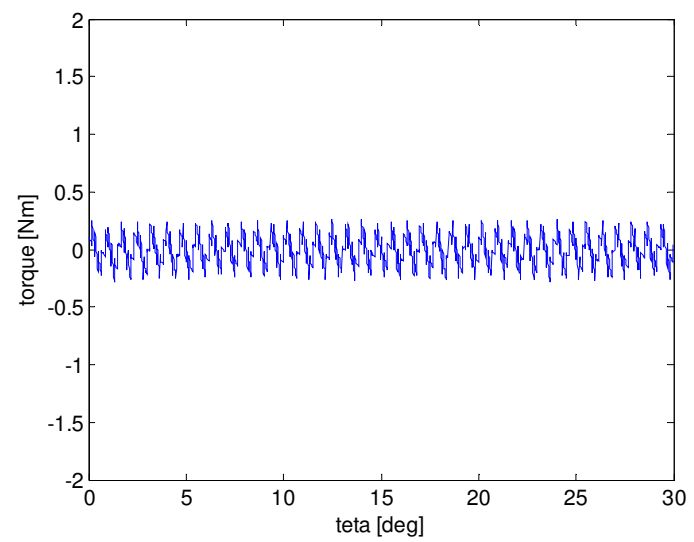

Fig. 6. Cogging torque versus rotor position (2D planeparallel approach, radial magnetization).

\section{Influence of BH characteristic of stator and rotor laminations}

The material properties of stator and rotor laminations can influence the values of the cogging torque of the generator. In order to prove this statement we considered two types of soft magnetic materials with different initial relative magnetic permeability values 500 and 1000 .

The computation results, Fig. 7, prove that the soft magnetic materials characterized by initial magnetic permeability value of 1000 generate cogging torque peak-to-peak values larger with roughly $16 \%$ than in the case of magnetic materials with an initial magnetic permeability value of 500 .

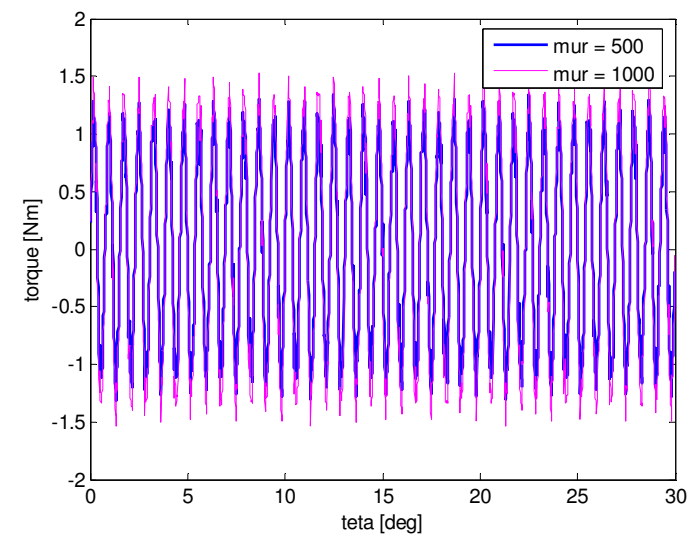

Fig. 7. Cogging torque versus rotor position (2D planeparallel approach, for different initial relative magnetic permeability values).

\section{3D magneto-static model of the machine}

The numerical values of the cogging torque ripples of the studied wind generator obtained by the 2D plane-parallel FEM approach, Fig. 3, are relatively high. A method for reducing these oscillations that was applied when building the experimental model of the studied wind generator consists in skewing the stator laminations with a properly chosen inclination angle (in our case an angle of $8.4^{\circ}$ was applied).

In the case of a machine with skewed slots, a 2D planeparallel FEM approach is not suitable any longer, a 3D FEM model being much more adequate.

Thus an elaborated 3D magneto-static model of the synchronous wind generator was developed, Fig. 8. This model is based on the magnetic scalar potential formulation that is governed by the following differential equation:

$$
\nabla \cdot[\mu \cdot \nabla \Phi]=\nabla \cdot \mathbf{B}_{\mathbf{r}}
$$

where $\Phi$ stands for the total magnetic scalar potential derived from the relation $\nabla \times \mathbf{H}=0$.

Based on the 3D computation model of the synchronous generator we obtained the chart of magnetic flux density on the computation domain, Fig. 9, and the cogging torque oscillations for different relative rotor/stator positions, Fig. 10. 
The cogging torque results were obtained after more than 48 hours of computation on a PC equipped with an Intel Core 2 Duo T7500 processor, with $2.2 \mathrm{GHz}$ frequency.

After the 3D FEM simulations, the obtained results were processed in order to eliminate the numerical noise due to the presence of only one layer of finite elements in the airgap region. In order to get higher accuracy results the proposed 3D model should be improved by the use of a more refined finite element discretization especially in the airgap region where three layers of finite elements are recommended. However such an improved 3D FEM model would require a more important hardware and software resources leading to a total computation time of several days or even weeks.

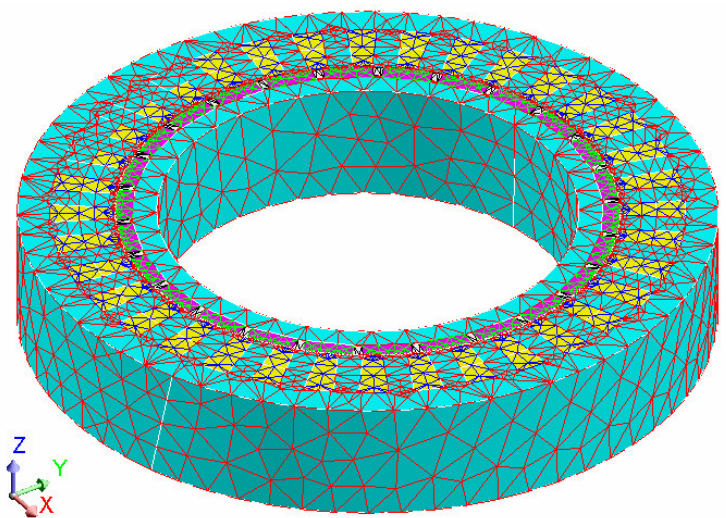

Fig. 8. 3D finite element computation domain and mesh of the studied machine.
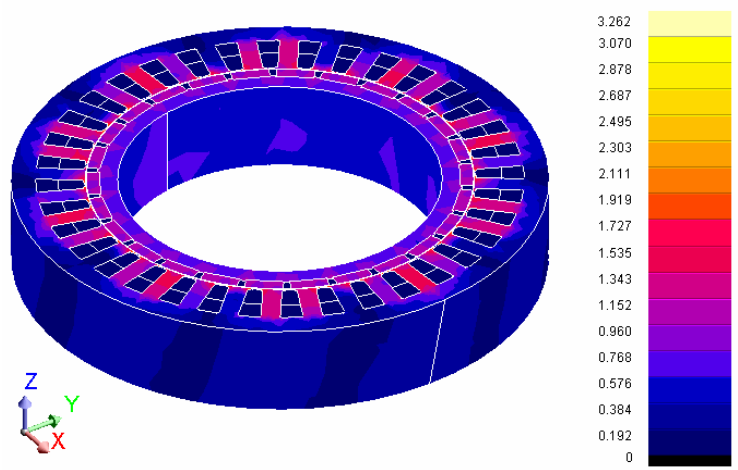

Fig. 9. Chart of magnetic flux density on the 3D finite element computation domain (B [T]).

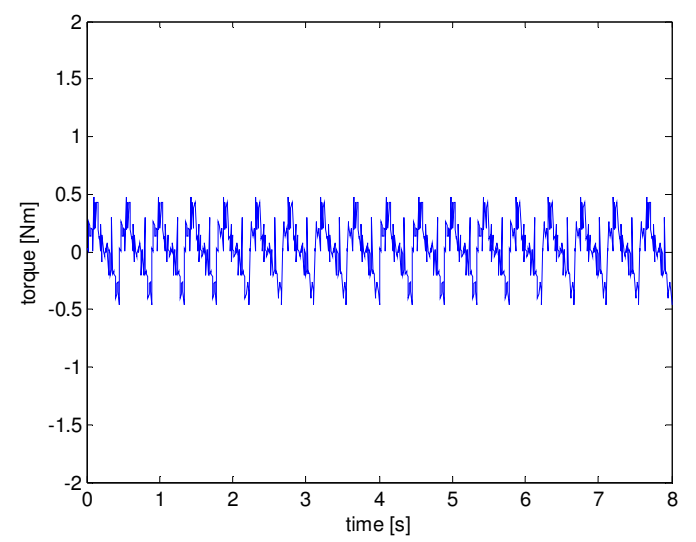

Fig. 10. Cogging torque variation versus rotor position in case of 3D approach (processed results).

\section{Experimental validation}

In order to validate the numerical simulation results we used an experimental facility consisting of the studied synchronous generator rotated with a slow velocity, Fig. 11, and a specialized torque transducer with automatic data recording system. The measurement data obtained in the ICPE-ME workshops were numerically processed in order to eliminate the friction torque influence on the cogging torque results.

A good agreement can be noticed between the peak-topeak values of the experimental results presented in Fig. 12, and of the numerical ones shown in Fig. 10.
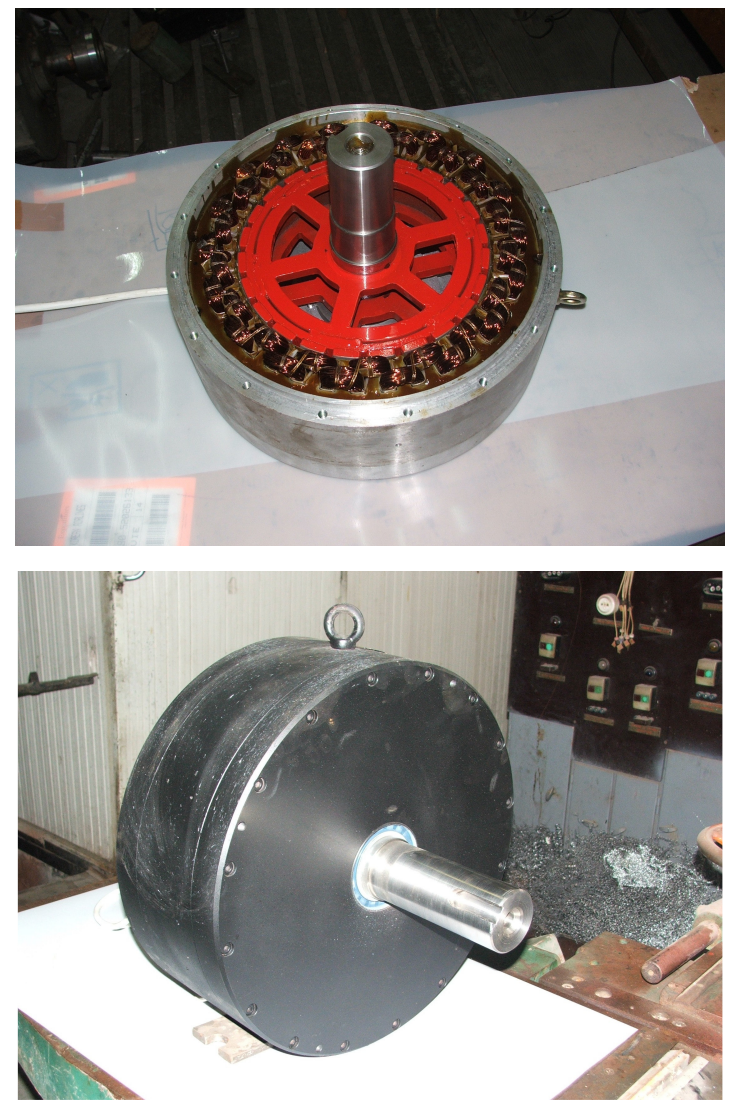

Fig. 11. Experimental model of studied multi-pole synchronous generator.

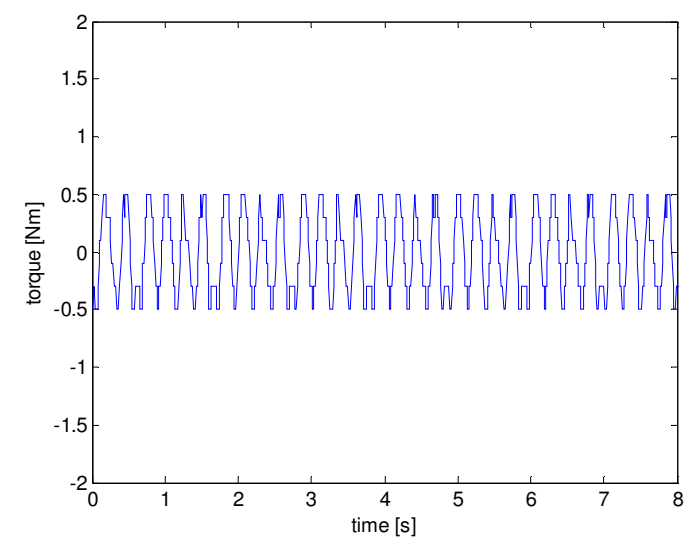

Fig. 12. Experimental measurement results of cogging torque (processed results). 


\section{Conclusion}

This study proves the utility and versatility of finite element based models in the optimal design of multi-pole permanent magnet synchronous generators by the prediction of the cogging torque oscillations, quantity that can influence the proper wind turbine operation.

The numerical results proved that the cogging torque results obtained in case of a generator with straight slots can be reduced by skewing the stator slots with a proper inclination angle.

The sensitivity analysis of the influence of magnetization orientation of permanent magnets proved that the cogging torque oscillations are more reduced in case of a radial orientation than in case of unidirectional orientation of permanent magnets.

The construction of magnetic armatures of the generator using soft magnetic materials with higher initial relative magnetic permeability leads to higher cogging torque values.

The 3D FEM results (peak-to-peak values) are in good agreement with the experimental measurements results obtained on a dedicated stand in the ICPE-ME workshops.

\section{Acknowledgment}

This paper is a result of a research activity carried out in the frame of PNCDI2 and CEEX research grants, and we take this occasion to thank the Romanian projects management institutions AMCSIT and CNMP for their support.

\section{References}

[1] A. Grauers, "Directly driven wind turbine generators", Proc. of the International Conference on Electrical Machines ICEM'96, Vigo (Spain), Sept. 1996, vol.2, pp. 417-422, 1996.

[2] P. Lampola, "Electromagnetic design of an unconventional directly driven permanent magnet wind generator", Proc. of the International Conference on Electrical Machines (ICEM'98), Istanbul, Turkey, 2-4 September, 1998, Vol. 3, pp. 1705-1710, 1998.

[3] P. Lampola, "Directly driven low speed PM generators for wind power applications", $\mathrm{PhD}$ Thesis, Acta Politechnica Scandinavica, Electrical engineering series, No.101, Espoo, 2000.

[4] M. Cistelecan, B. Cosan, M. Popescu, "Tooth concentrated fractional windings for low speed three phase a.c. machines", accepted paper to be presented at ICEM'06, Chania, Greece, Sept. 2006.

[5] M. Popescu, M. Cistelecan, L. Melcescu, M. Covrig, "Low speed directly driven permanent magnet synchronous generators for wind energy applications", Proc. of IEEE International Conference on Clean Electrical Power, ICCEP 2007, Capri, Italy, 2007. 